\title{
Positron Emission Mammography with Multiple Angle Acquisition
}

\author{
Mark F. Smith, Member, IEEE, Stan Majewski and Raymond R. Raylman, Member, IEEE
}

\begin{abstract}
Positron emission mammography (PEM) of F-18 fluorodeoxyglucose (FDG) uptake in breast tumors with dedicated detectors typically has been accomplished with two planar detectors in a fixed position with the breast under compression. The potential use of PEM imaging at two detector positions to guide stereotactic breast biopsy has motivated us to use PEM coincidence data acquired at two or more detector positions together in a single image reconstruction. Multiple angle PEM acquisition and iterative image reconstruction were investigated using point source and compressed breast phantom acquisitions with 5, 9, 12 and $15 \mathrm{~mm}$ diameter spheres and a simulated tumor:background activity concentration ratio of 6:1. Image reconstruction was performed with an iterative MLEM algorithm that used coincidence events between any two detector pixels on opposed detector heads at each detector position. This present study compared two acquisition protocols: 2 angle acquisition with detector angular positions of -15 and +15 degrees and 11 angle acquisition with detector positions spaced at 3 degree increments over the range -15 to +15 degrees. Three-dimensional image resolution was assessed for the point source acquisitions, and contrast and signal-to-noise metrics were evaluated for the compressed breast phantom with different simulated tumor sizes. Radial and tangential resolutions were similar for the two protocols, while normal resolution was better for the 2 angle acquisition. Analysis is complicated by the asymmetric point spread functions. Signalto-noise vs. contrast tradeoffs were better for 11 angle acquisition for the smallest visible $9 \mathrm{~mm}$ sphere, while tradeoff results were mixed for the larger and more easily visible $12 \mathrm{~mm}$ and $15 \mathrm{~mm}$ diameter spheres. Additional study is needed to better understand the performance of limited angle tomography for PEM. PEM tomography experiments with complete angular sampling are planned.
\end{abstract}

\section{INTRODUCTION}

$\mathrm{F}$ unctional metabolic imaging of breast tissue and carcinomas with F-18 fluorodeoxyglucose (FDG) has been performed with positron emission tomography (PET) [1, 2] and now positron emission mammography (PEM) with dedicated detectors is being investigated as a way of imaging increased FDG uptake in breast tumors [3, 4]. These special purpose detectors can improve coincidence detection sensitivity by being positioned near the breast and by

This work is supported by the Office of Biological and Environmental Research of the Office of Science of the U.S. Department of Energy and by grant 1 R21 CA82752-01 from the National Cancer Institute. The Southeastern Universities Research Association (SURA) operates the Thomas Jefferson National Accelerator Facility for the United States Department of Energy under contract DE-AC05-84ER40150.

M. F. Smith (telephone 757-269-5539; mfsmith@jlab.org) and S. Majewski (majewski@jlab.org) are with the Thomas Jefferson National Accelerator Facility, Newport News, VA 23606 USA.

R. R. Raylman (rraylman@wvu.edu) is with the Department of Radiology, Robert C. Byrd Health Sciences Center, West Virginia University, Morgantown, WV 26506 USA. eliminating high attenuation raypaths through the torso. With smaller crystals than in general purpose whole body PET scanners, they have the potential to improve detection, visualization and localization of small tumors.

PEM acquisition typically has been accomplished with two planar detectors in a fixed position with the breast under compression, though other imaging devices and geometries also have been proposed [5-10]. Recently we have investigated breast imaging with a single detector position [11] and the potential use of PEM imaging at two detector positions to guide stereotactic breast biopsy [12]. This latter work has motivated us to consider how the acquired PEM coincidence data from two or more detector positions could be used together in image reconstruction. In this contribution we investigate multiple angle PEM acquisition and image reconstruction with experimental PEM acquisitions of point sources and a compressed breast phantom. Three-dimensional image resolution was assessed for the point source acquisitions, and contrast and signal-to-noise metrics were evaluated for the compressed breast phantom with different simulated tumor sizes.

\section{METHODS}

\section{A. Data acquisition}

Experimental acquisitions were made with point sources and a compressed breast phantom with simulated spherical tumors in order to evaluate the resolution, contrast and signalto-noise characteristics for two acquisition protocols. For the first protocol, coincidence data were acquired with the detector at two orientations: +15 and -15 degrees with respect to the plane containing the cross of beads or the plane containing the compressed breast (Figure 1). For the second protocol, coincidence data were acquired with the PEM detector at 11 angular positions spaced at a 3 degree increment from -15 degrees to +15 degrees. Rotation was accomplished by placing the point sources or phantom on a rotation stage, whose axis of rotation was parallel to the detector surfaces and midway between the detectors (Figure 2). For clinical acquisitions the compressed breast would be stationary and the PEM detector would rotate.

List-mode coincidence data were acquired with a dual detector gadolinium oxyorthosilicate (GSO) PEM system. Each detector head was built with a $30 \times 30$ array of $3.1 \times 3.1$ $\mathrm{x} 10 \mathrm{~mm}^{3}$ GSO crystals (Hitachi, Inc.) with a crystal pitch of $3.3 \mathrm{~mm}$; the field of view was $10 \mathrm{~cm} \times 10 \mathrm{~cm}$. A one $\mathrm{cm}$ thick acrylic light guide coupled the crystals to a $4 \times 4$ array of Hamamatsu R5900-C8 position-sensitive photomultiplier tubes. Data acquisition was under the control of software developed using the KMAX development system 


\section{DETECTOR ORIENTATIONS}
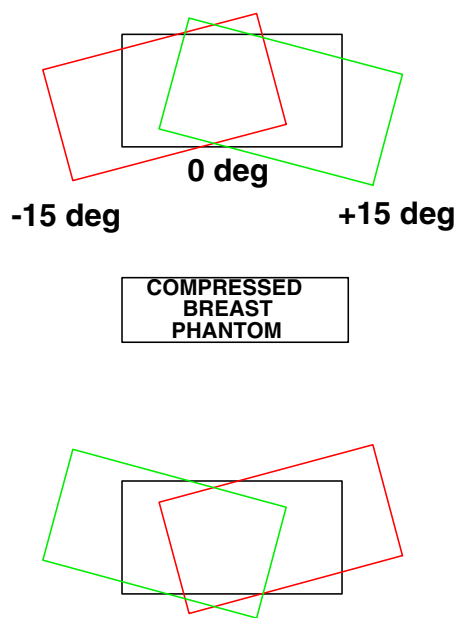

Figure 1. Diagram of the PEM detector orientations with respect to the compressed breast phantom.

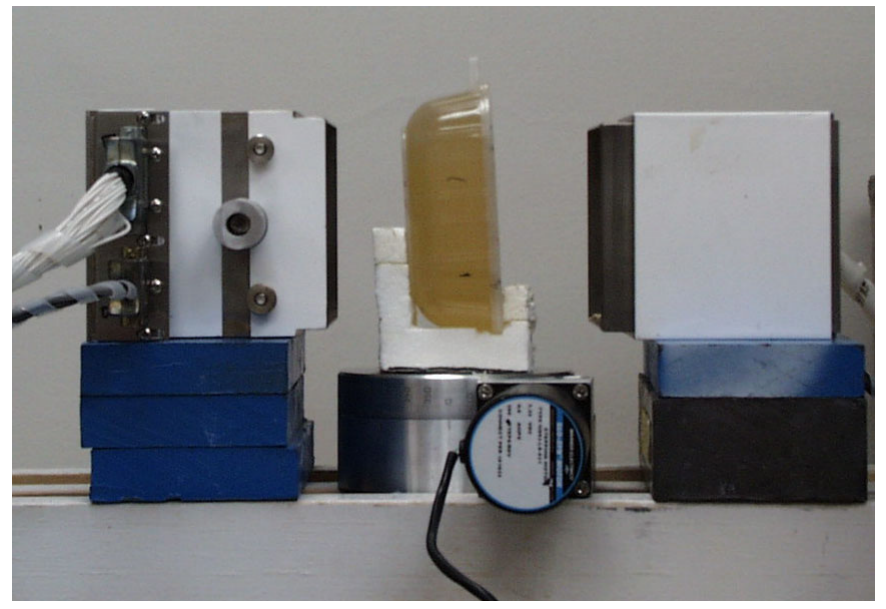

Figure 2. PEM detectors (right and left) and the gelatin compressed breast phantom on a rotation stage (center).

(Sparrow, Inc.) running on an Apple Macintosh G4 computer. For a complete system description see [11].

A series of acquisitions was made with ceramic beads, each approximately $2 \mathrm{~mm}$ in diameter, that had been soaked for 5 $\min$ in a $7.4 \mathrm{MBq} / \mathrm{ml}(200 \mu \mathrm{Ci} / \mathrm{ml})$ solution of F-18. Nine beads were arranged in a cross pattern, with four beads $2 \mathrm{~cm}$ from the center bead and an additional four beads $4 \mathrm{~cm}$ from the center bead. The plane containing the cross was parallel to the detector head surfaces at a rotation angle of zero degrees. List mode data were acquired for two minutes at each of -15 and +15 degree camera positions, and then for 30 $\mathrm{sec}$ at each of 11 angles with camera positions from -15 to +15 degrees. The separation between the surfaces of the detector heads was $20 \mathrm{~cm}$, modeling the separation for stereotactic core biopsy.

A compressed breast phantom was made by forming a 4 $\mathrm{cm}$ thick gelatin block with an F-18 activity concentration of $3.40 \mathrm{kBq} / \mathrm{ml}(0.092 \mu \mathrm{Ci} / \mathrm{ml})$. Four spheres with diameters 5, 9, 12 and $15 \mathrm{~mm}$ were filled with an F-18 solution and placed within the gelatin block. The activity concentration in the spheres was $20.4 \mathrm{kBq} / \mathrm{ml}(0.55 \mu \mathrm{Ci} / \mathrm{ml})$, for a simulated tumor:background activity concentration ratio of $6: 1$. The phantom was positioned midway between the detectors, which again were $20 \mathrm{~cm}$ apart. There were two acquisition sequences, each for a total duration of five minutes. For the first acquisition, data were acquired for 150 seconds at +15 and -15 degrees (Figure 1). For the second acquisition, data were acquired for $27 \mathrm{sec} /$ angle at each of 11 equally spaced angles ( 3 degree increment) between -15 and +15 degrees

In order to determine the center of rotation of the imaging system, coincidence data were acquired for a line source filled with F-18 with the PEM detector at 18 angles (20 degree increment) over 360 degrees. The center of rotation shift was determined to be $1 \mathrm{~mm}$ by analyzing several test images reconstructed using the iterative algorithm described in the following section.

\section{B. Image reconstruction}

Coincidence events along each line of response (LOR) were tallied from the list-mode data and then images were reconstructed using the maximum-likelihood expectationmaximization (MLEM) algorithm [13]. A single-angle implementation of MLEM for PEM [14] was enhanced to allow image reconstruction using LORs from different detector orientations simultaneously. Note that for the acquisition geometry of this experiment, coincidence data at just a single detector position yields LORs spanning an angular range of $2 \times \tan (10 / 20)=54$ degrees in a plane perpendicular to the rotation axis for a point on the rotation axis.

An energy window of 400-700 $\mathrm{keV}$ was used and the voxel size in the reconstructed images was $1.65 \mathrm{~mm}$, which is half the pitch of the detector pixels. The three-dimensional image volume was a cube 60 voxels on a side. Ten iterations were performed for image reconstructions using coincidence data from: 1) -15 degrees and +15 degrees detector orientations only and 2) the 11 equally spaced angles between -15 and +15 degrees. In addition, some illustrative images were reconstructed using coincidence data from -15 degrees only and +15 degrees only.

\section{Image analysis}

For the point source phantom, resolution was measured in three orthogonal directions: radially and tangentially in the plane containing the point sources and normal to this plane. A Gaussian curve was fit to profiles through the point sources and the full-width at half-maximum (FWHM) was computed.

For the breast phantom, regions of interest (ROIs) were drawn within the visible tumors $(\mathrm{T})$ and in adjacent background regions (B). The mean and standard deviation of the voxel values within each ROI were computed. Tumor contrast was calculated using the formula $\mathrm{C}=(\mathrm{T}-\mathrm{B}) / \mathrm{B}$ and the signal-to-noise ratio was computed using the formula $\mathrm{SNR}=(\mathrm{T}-\mathrm{B}) / \sigma(\mathrm{B})$. The analysis was automated so that these quantities were computed at each iteration. 


\section{A. 2 Angles}
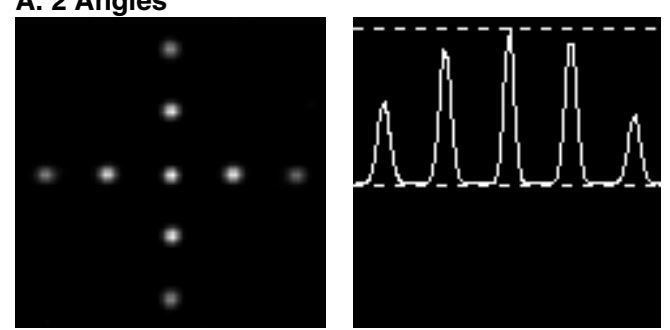

\section{B. 11 Angles}
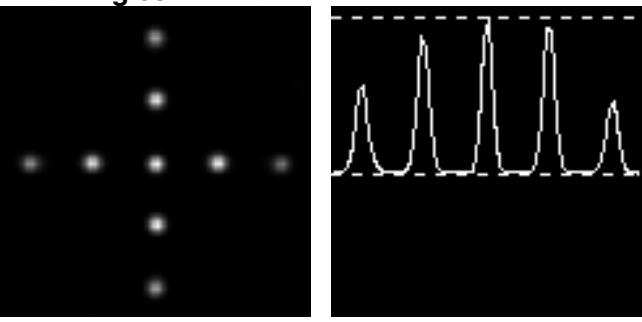

Figure 3. Reconstructed image slices through the cross arrangement of the point sources for (a) two angle (-15 and +15 degrees) and (b) multiple angle (11 angles between -15 and +15 degrees) PEM acquisitions. The profiles are through the central row of point sources.

\section{A. 2 Angles}
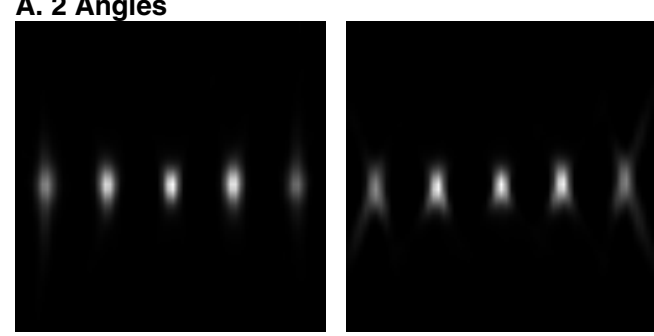

\section{B. 11 Angles}
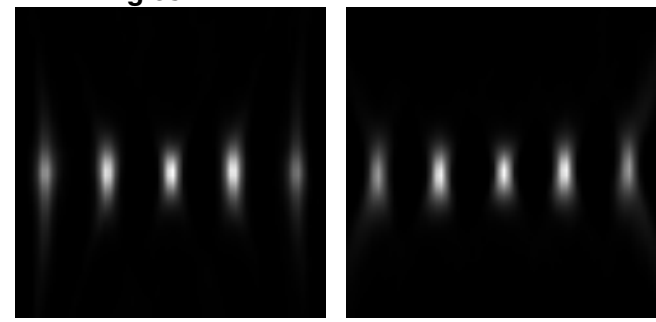

Figure 4. Reconstructed image slices of the point sources for (a) two angle ( -15 and +15 degrees) and (b) multiple angle (11 angles between -15 and +15 degrees) PEM acquisitions. (Left) image plane normal to the cross pattern and parallel to the rotation axis and (right) image plane normal to the cross pattern and normal to the rotation axis.
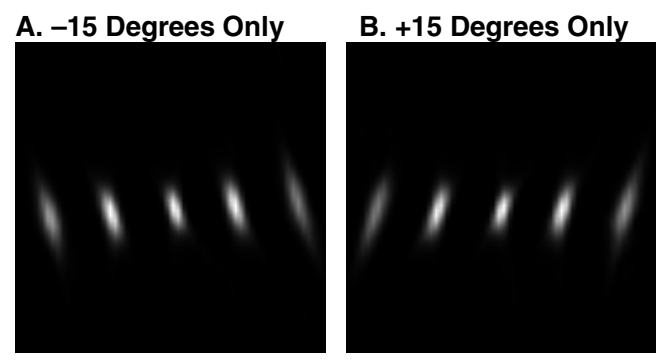

Figure 5. Reconstructed image slices of the point sources for (a) single angle ( -15 degrees only) and (b) single angle ( +15 degrees only) PEM acquisitions. These image slices are from planes normal to the cross pattern and normal to the rotation axis.
A

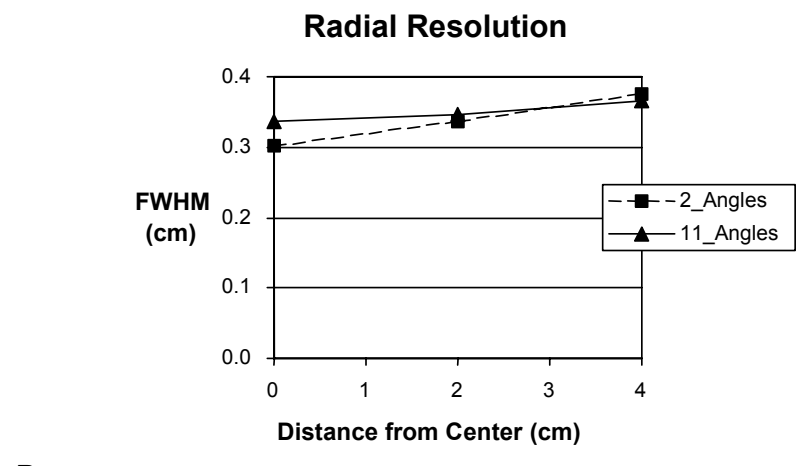

B

Tangential Resolution

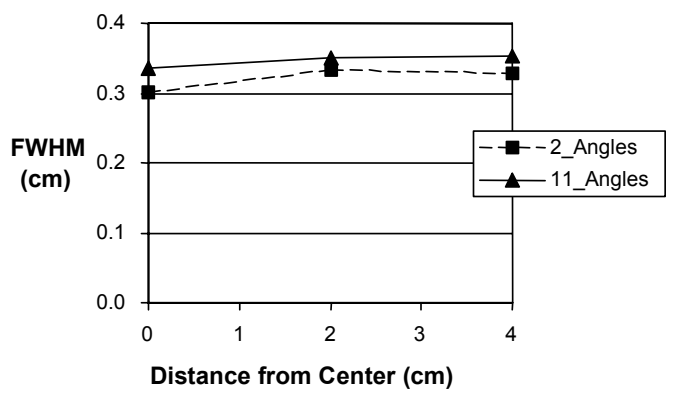

C

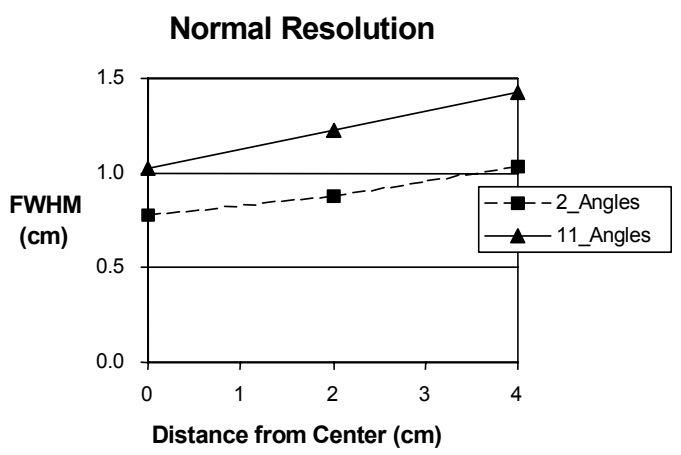

Figure 6. Point source resolutions (FWHM) (a) radial in the plane containing the sources, (b) tangential in the plane containing the sources and (c) normal to the plane containing the point sources (i.e. normal to the detector face at 0 degree rotation angle).

\section{RESULTS}

\section{A. Point source phantom}

The point source resolutions stabilized after a few iterations and results are presented here for six iterations. Image slices through the plane containing the point sources are similar for the 2 angle and 11 angle acquisitions (Figure 3 ) and this is reflected in similar values for radial and tangential resolution (Figure 6a,b). Images normal to the plane containing the cross are also similar (Figure 4), though the resolution normal to the cross is poorer for the 11 angle acquisitions (Figure 6c) and streak artifacts are more obvious for the 2 angle acquisitions (Figure 4a). Image reconstructions using coincidence data from only a single 


\section{A. 2 angle}
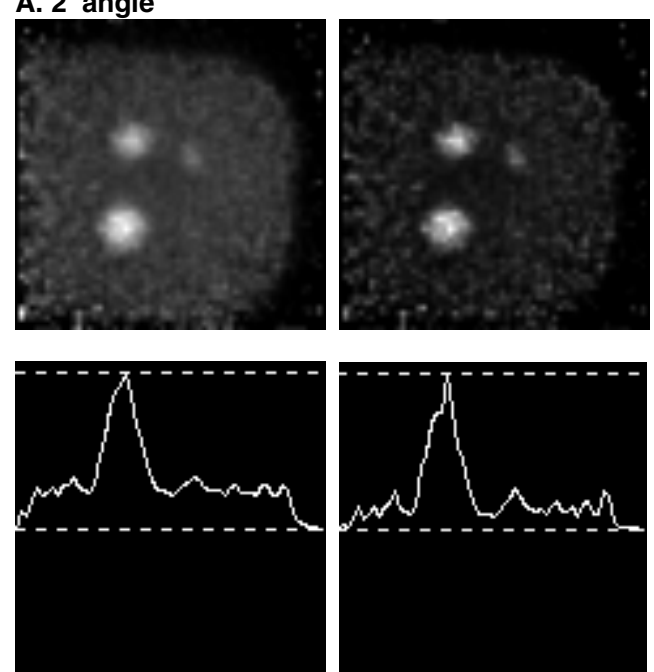

\section{B. 11 angle}
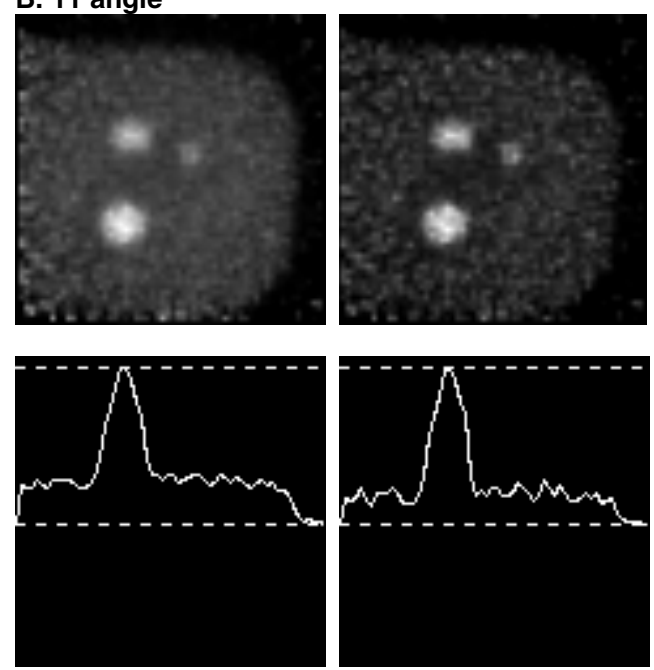

Figure 7. Reconstructed image slice through the simulated breast phantom tumors for (a) two angle ( -15 and +15 degrees) and (b) multiple angle (11 angles between -15 and +15 degrees) PEM acquisitions. The image on the left is from two iterations and the image on the right from six iterations of the MLEM algorithm. Horizontal profiles through the largest $15 \mathrm{~cm}$ diameter sphere are shown. The 12 and $9 \mathrm{~mm}$ diameters spheres can be seen easily but the $5 \mathrm{~mm}$ diameter sphere is not visible.

angle show that resolution is degraded in a direction normal to the detector surfaces (Figure 5).

\section{B. Compressed breast phantom}

Slices through the reconstructed image volumes show that the 15, 12 and $9 \mathrm{~mm}$ diameter spheres are easily visible (Figure 7), while the $5 \mathrm{~mm}$ diameter sphere cannot be visualized. The effect of blurring normal to the detectors can be seen in Figures 8 and 9. Some angular streak artifacts can be seen in Figure 9, which are slices perpendicular to the rotation axis and normal to the detector surface at 0 degrees.

A plot of the signal-to-noise ratio vs. contrast as a function of iteration is displayed in Figure 10. For the $15 \mathrm{~mm}$ and 12 $\mathrm{mm}$ diameter spheres contrast increases with iteration while SNR increases then decreases for both 2 angle and 11 angle acquisitions. The greatest SNR for the $15 \mathrm{~mm}$ diameter sphere is achieved with 11 angle acquisition while

\section{A. 2 angle}
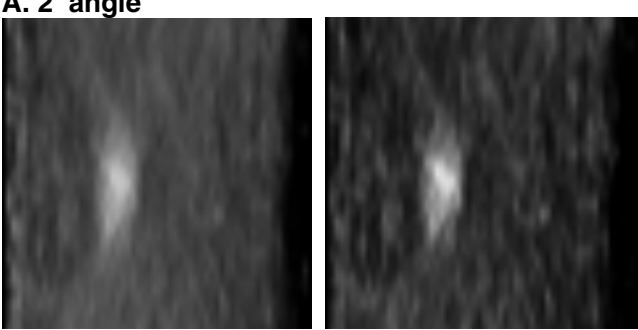

\section{B. 11 angle}
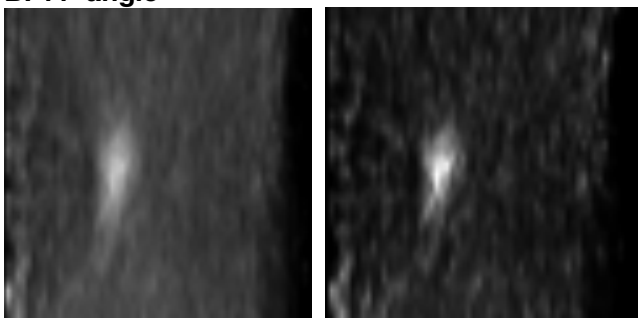

Figure 8. Reconstructed image slices through the $12 \mathrm{~mm}$ diameter sphere for (a) two angle ( -15 and +15 degrees) and (b) multiple angle (11 angles between -15 and +15 degrees) PEM acquisitions. The image slice is parallel to the rotation axis and normal to the detector surface at 0 degrees. (Left) two iterations and (right) six iterations.

\section{A. 2 angle}
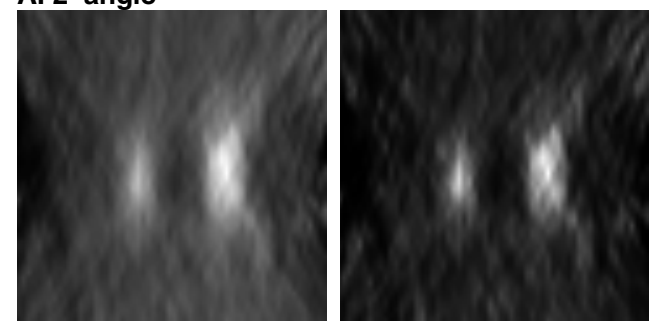

\section{B. 11 angle}
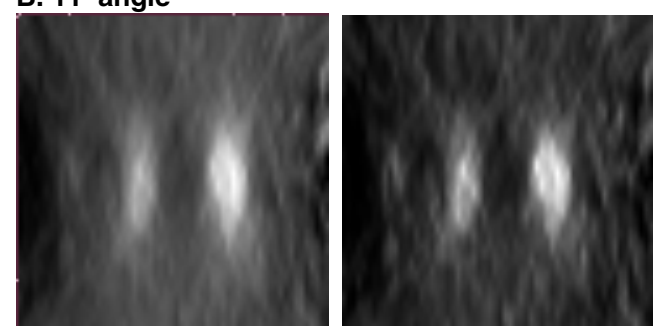

Figure 9. Reconstructed image slices through the 12 and $15 \mathrm{~mm}$ diameter spheres for (a) two angle ( -15 and +15 degrees) and (b) multiple angle (11 angles between -15 and +15 degrees) PEM acquisitions. The image slice is normal to the rotation axis and normal to the detector surface at 0 degrees. (Left) two iterations and (right) six iterations.

\section{SNR vs Contrast}

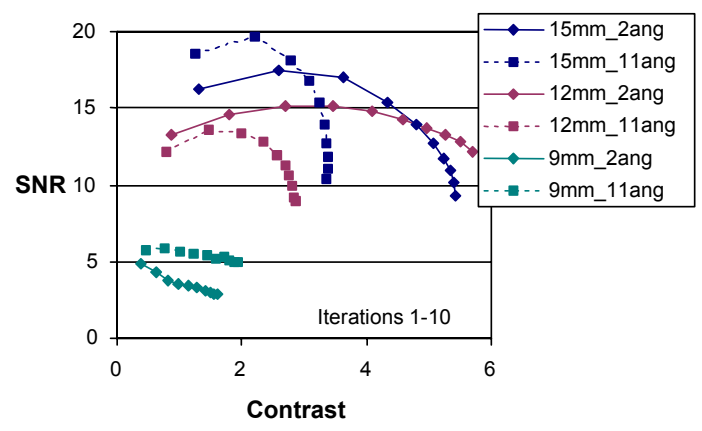

Figure 10. Signal-to-noise ratio vs. contrast as a function of iteration for the different spheres in the compressed breast phantom. The value at each iteration is indicated; iteration 1 has the least contrast for each curve. 
the SNR is greatest for the $12 \mathrm{~mm}$ diameter sphere with 2 angle acquisition.

The variation of SNR and contrast is simpler for the smaller $9 \mathrm{~mm}$ diameter sphere, where for both 2 angle and 11 angle aquisitions the SNR trend is decreasing with iteration while the contrast increases. At equal contrast, the SNR is always greater for the 11 angle acquisition than for the 2 angle acquisition.

\section{DISCUSSION}

Limited angle tomography can be accomplished using coincidence data from two or more PEM detector positions. At each detector position there is a range of angles for which LORs pass through the source region, since LORs between any two detector pixels on opposite detector heads are used in image reconstruction. Sampling of the source region is more dense and complete as the number of detector positions increases.

The tangential and radial resolutions are about the same for the two angle ( -15 and +15 degrees) and eleven angle (uniform at a 3 degree increment between -15 and +15 degrees) acquisitions. The normal resolution is less for the 2 angle acquisition than for the 11 angle acquisition. The reason for this result can be seen in Figure 4. For the 2 angle reconstruction, there are tails that can be seen at approximately +15 and -15 degrees from the vertical, whereas for the 11 angle acquisition there are tails at each of the 11 angles that fuse into a continuous blur. This blur extends in the vertical direction, causing normal resolution at the center of the point sources to be worse for the 11 angle case than for the 2 angle case. Caution must be taken when interpreting FWHM values for these asymmetric point spread functions.

The signal-to-noise vs. contrast tradeoffs for the 11 angle acquisition have an advantage over those for the 2 angle acquisition for the smallest visible $9 \mathrm{~mm}$ diameter sphere (Figure 10). For the two larger spheres, the 2 angle acquisition has the advantage for the $12 \mathrm{~mm}$ sphere, while the tradeoff curves cross for the $15 \mathrm{~mm}$ tumor. Additional work is needed to better understand these tradeoffs for different simulated tumor sizes.

With either acquisition protocol, resolution normal to the detectors is poor. Stereotactic localization of point sources by projecting rays from their centers at two different views has an accuracy of approximately one $\mathrm{mm}[12]$ and thus appears preferable to localization based on a three-dimensional limited angle tomographic reconstruction. This is probably due to the fact that stereotactic localization relies on the strong assumptions that the same source region of increased activity concentration can be identified in image reconstruction from two different angular views and that the focal source region is localized in a compact shape. These constraints are not built into the implementation of the iterative reconstruction algorithm, though better reconstructions might result if a suitable prior were incorporated into the iterative algorithm.

\section{CONCLUSIONS}

Multiple angle PEM acquisition and iterative image reconstruction have been investigated using point source and compressed breast phantoms. This particular study compared 2 angle and 11 angle acquisitions over a detector range of -15 to +15 degrees. Radial and tangential resolutions were similar for the two methods, while normal resolution was better for the 2-angle acquisition. The point spread functions were asymmetric for both cases, however. For the smallest visible $9 \mathrm{~mm}$ sphere signal-to-noise vs. contrast tradeoffs were better for 11 angle acquisition, while tradeoff results were mixed for the larger and more easily visible $12 \mathrm{~mm}$ and $15 \mathrm{~mm}$ diameter spheres. Additional study is needed to better understand the performance of limited angle tomography for PEM. Future work will include PEM tomography with complete angular sampling.

\section{REFERENCES}

[1] R. L. Wahl, R. L. Cody, G. D. Hutchins, and E. E. Mudgett, "Primary and metastatic breast carcinoma: initial clinical evaluation with PET with the radiolabeled glucose analogue 2-[F-18]-fluoro-2-deoxy-D-glucose," Radiology, vol. 179, pp. 765-770, 1991.

[2] L. P. Adler, J. P. Crowe, N. K. Al-Kaisi, and J. L. Sunshine, "Evaluation of breast masses and axillary lymph nodes with [F-18] 2-deoxy-2-fluoro-Dglucose PET," Radiology, vol. 187, pp. 743-750, 1993.

[3] I. Weinberg, S. Majewski, A. Weisenberger, A. Markowitz, L. Aloj, L. Majewski, D. Danforth, J. Mulshine, K. Cowan, J. Zujewski, C. Chow, E. Jones, V. Chang, W. Berg, and J. Frank, "Preliminary results for positron emission mammography: real-time functional breast imaging in a conventional mammographic gantry," Eur. J. Nucl. Med., vol. 23, pp. 804$806,1996$.

[4] K. Murthy, M. Aznar, C. J. Thompson, A. Loutfi, R. Lisbona, and J. H. Gagnon, "Results of preliminary clinical trials of the positron emission mammography system PEM-I: a dedicated breast imaging system producing glucose metabolic images using FDG," J. Nucl. Med., vol. 41, pp. 1851$1858,2000$.

[5] W. W. Moses, T. F. Budinger, R. H. Huesman, and S. E. Derenzo, "PET camera designs for imaging breast cancer and axillary node involvement," $J$. Nucl. Med., vol. 36, pp. 69P, 1995.

[6] G. D. Hutchins and A. J. Simon, "Evaluation of prototype geometries for breast imaging with PET radiopharmaceuticals," J. Nucl. Med., vol. 36, pp. 69P-70P, 1995.

[7] R. Freifelder and J. S. Karp, "Dedicated PET scanners for breast imaging," Phys. Med. Biol., vol. 42, pp. 2463-2480, 1997.

[8] R. H. Huesman, G. J. Klein, W. W. Moses, J. Qi, B. W. Reutter, and P. R. G. Virador, "List-mode maximum-likelihood reconstruction applied to positron emission mammography (PEM) with irregular sampling," IEEE Trans. Med. Imaging, vol. 19, pp. 532-537, 2000.

[9] H. Baghaei, W.-H. Wong, J. Uribe, H. Li, N. Zhang, and Y. Wang, "Breast cancer imaging studies with a variable field of view PET camera," IEEE Trans. Nucl. Sci., vol. 47, pp. 1080-1084, 2000.

[10] N. K. Doshi, R. W. Silverman, Y. Shao, and S. R. Cherry, "maxPET: a dedicated mammary and axillary region PET imaging system for breast cancer," IEEE Trans. Nucl. Sci., vol. 48, pp. 811-815, 2001.

[11] R. R. Raylman, S. Majewski, R. Wojcik, A. G. Weisenberger, B. Kross, V. Popov, and H. A. Bishop, "The potential role of positron emission mammography for detection of breast cancer. A phantom study," Med. Phys., vol. 27, pp. 1943-1954, 2000.

[12] R. R. Raylman, S. Majewski, A. G. Weisenberger, V. Popov, R. Wojcik, B. Kross, J. S. Schreiman, and H. A. Bishop, "Positron emission mammography-guided breast biopsy," J. Nucl. Med., vol. 42, pp. 960-966, 2001.

[13] K. Lange and R. Carson, "EM reconstruction algorithms for emission and transmission tomography," J. Comput. Assist. Tomogr., vol. 8, pp. 306-316, 1984.

[14] M. F. Smith, S. Majewski, A. G. Weisenberger, D. A. Kieper, R. R. Raylman, and T. G. Turkington, "Analysis of factors affecting positron emission mammography (PEM) image formation," IEEE Trans. Nucl. Sci., in press. 